\title{
THE NUMERICAL SIMULATION OF LIQUID SLOSHING IN MICROGRAVITY
}

\author{
Roel Luppes*, Joop A. Helder and Arthur E.P. Veldman \\ *University of Groningen \\ Institute of Mathematics and Computing Science \\ P.O. Box 800, 9700 AV Groningen, The Netherlands \\ e-mail: r.luppes@math.rug.nl
}

Key words: Free-Surface Flows, Microgravity, Solid-Liquid Interaction, Sloshsat FLEVO, Numerical Simulations

\begin{abstract}
The subject of study is the influence of sloshing liquid on board spacecraft and satellites. Experiments have been carried out with the mini satellite Sloshsat FLEVO, containing a partially filled water tank, in an orbit around earth. These experiments were supported by a computational model for 3D incompressible free-surface flow, including capillary surface physics and coupled solid-liquid interaction dynamics. Experimental results and numerical simulations are compared. The obtained frequencies in angular velocities are reasonably comparable at various satellite manoeuvres. The damping of nutation amplitudes observed in the simulations is too large, as a result of additional diffusion in the computational model. At low rotational rates and small-scale liquid motion, capillary effects are important for the satellite motion and the observed damping of manoeuvreinduced oscillations. In case of large-scale liquid motion, such as flat-spin manoeuvres, capillary forces are less important.
\end{abstract}

\section{INTRODUCTION}

In this study, the influence of sloshing liquid on board spacecraft and satellites is investigated. With the increasing amount of liquid on board spacecraft, liquid management and its influence on the overall spacecraft dynamics is becoming increasingly important. The influence of sloshing liquid may hamper critical manoeuvres in space, such as the docking of liquid-cargo vehicles or the pointing of observational satellites. Severe problems with sloshing liquid in space have been reported. For example, the NEAR satellite (Strikwerda et al. ${ }^{1}$, NEAR Anomaly Review Board ${ }^{2}$ ) went into safety mode because of an unexpected reaction that was possibly due to propellant slosh after an orbital manoeuvre, causing one year delay of the project. During the first lunar landing with Apollo 11, a dramatic propellant slosh problem occurred at the end of a yaw manoeuvre. Hence, much additional thruster activity was needed for course corrections and finally the Eagle landed at a different spot than originally planned (The Lunar Surface journal ${ }^{3}$ ). 


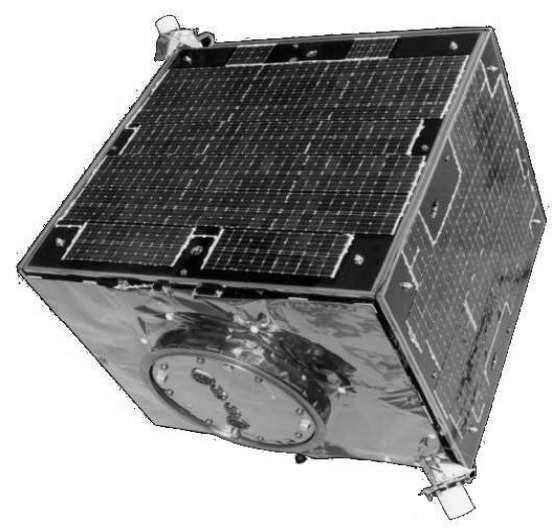

Figure 1: The Sloshsat FLEVO satellite.

The experimental study of the interaction between liquid sloshing and spacecraft dynamics, by means of droptower or parabolic flight, is difficult because of the short duration of terrestrial free fall. Therefore, experiments have been carried out recently with the satellite 'Sloshsat FLEVO' in an orbit around earth. This mini satellite has been built by the Dutch Aerospace Laboratory (NLR) $)^{4}$. A cylindrical shaped fluid tank with hemispherical ends, with a volume of 86.9 liters, is positioned inside the satellite. This tank is partly filled with $33.5 \mathrm{~kg}$ of distilled water, which could freely slosh during the experiments. The experiments were controlled by means of 12 thrusters that were fed with nitrogen gas. During the experiments, several relevant quantities for the Sloshsat motion were measured, such as the angular velocity, the linear acceleration and the pressure inside the fuel tank. A description of the measurement instruments on board as well as various other Sloshsat data is given by Vreeburg ${ }^{5-7}$. A description of the technological equipment inside Sloshsat is given by Prins ${ }^{8}$. In Figure 2, a schematic view inside Sloshsat is depicted.

The experiments with Sloshsat were supported by a theoretical/computational model based on the Navier-Stokes equations for 3D incompressible free-surface flow. This model includes capillary surface physics as well as coupled solid-liquid interaction dynamics, which is described in the next section. By means of such a model, more insight can be gained in the relevant physics that plays a part in the interaction between liquid sloshing and overall spacecraft dynamics. In the absence of gravity, capillary effects at the free liquid surface, such as surface tension and wetting characteristics, are dominating liquid motion. An overview of the relevant physics and modelling techniques for sloshing liquids is given by Vreeburg and Veldman ${ }^{9}$. Specific scientific areas in liquid dynamics where knowledge is lacking are contact-line behaviour and damping.

The present Computational Fluid Dynamics (CFD) code Comflo is the successor of the model that was used in the early 1980's as a support to experiments on board Spacelab (Veldman and Vogels ${ }^{10}$ ). Comflo is also used for maritime, industrial and offshore freesurface flow applications (Kleefsman et al. ${ }^{11}$, Fekken ${ }^{12}$ ). 


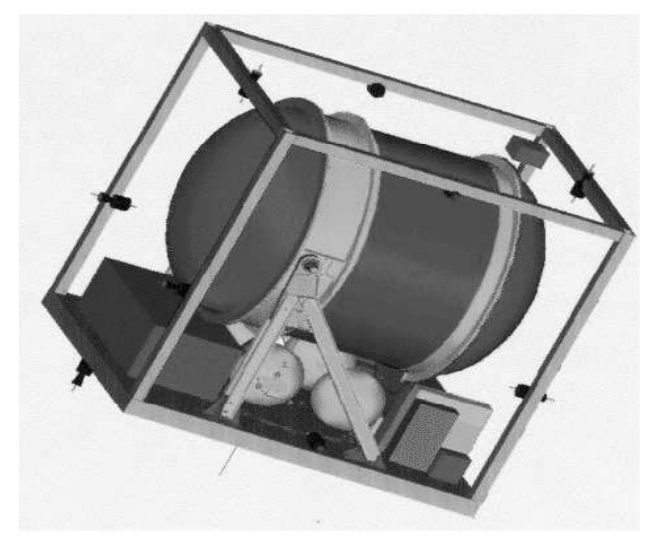

Figure 2: A view inside the Sloshsat satellite.

\section{THE MATHEMATICAL MODEL}

\subsection{Liquid dynamics}

The fluid flow inside Sloshsat can be described by means of the equations of motion, describing transport of e.g. mass, momentum and energy. For an incompressible fluid with constant viscosity, conservation of mass is given by the continuity equation

$$
\nabla \cdot \mathbf{u}=0
$$

and conservation of momentum in three directions is given by

$$
\frac{\partial \mathbf{u}}{\partial t}+(\mathbf{u} \cdot \nabla) \mathbf{u}=-\frac{1}{\rho} \nabla p+\frac{\mu}{\rho} \Delta \mathbf{u}+\boldsymbol{F}+\boldsymbol{f} .
$$

In these equations, $\mathbf{u}$ denotes the velocity of the fluid, $p$ denotes the pressure and $\rho$ and $\mu$ denote the fluid density and viscosity, respectively. The vectors $\boldsymbol{F}$ and $\boldsymbol{f}$ represent an additional acceleration due to an external force (such as gravity, which is however neglected in low-gravity situations) and a virtual body force, respectively. The latter is a consequence of the use of an inertial reference frame in the mathematical model for solidbody dynamics (Section 2.2), whereas equations (1) and (2) are relative to a reference frame that is fixed to the moving body. Equations (1) and (2) are often referred to as the Navier-Stokes equations for incompressible flow.

Boundary conditions are required at the solid tank wall and at the free surface. A schematic overview of the boundary conditions is given in Figure 3. The no-slip boundary condition for viscous flow is applied at the tank wall, i.e. $\mathbf{u}=0$, stating that the solid boundary is impermeable and that the fluid sticks to the wall due to viscous effects. Balancing the forces at the free surface in tangential $(\boldsymbol{t})$ and normal $(\boldsymbol{n})$ direction results in a tangential free-surface condition

$$
\mu\left(\frac{\partial u_{n}}{\partial \boldsymbol{t}}+\frac{\partial u_{t}}{\partial \boldsymbol{n}}\right)=0,
$$




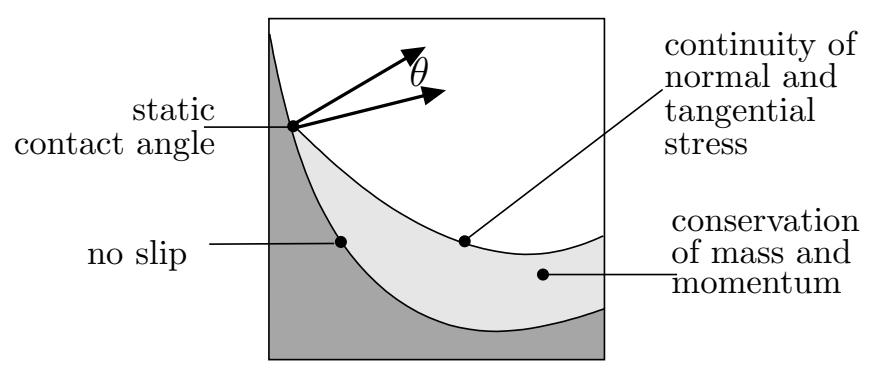

Figure 3: Schematic overview of the liquid model. Dark and light shading represent solid body and liquid, respectively.

which states that the air exerts no tangential stress on the fluid, and a normal condition

$$
-p+2 \mu \frac{\partial u_{n}}{\partial \boldsymbol{n}}=-p_{0}+\sigma \kappa
$$

which is however often simplified to $p=p_{0}-\sigma \kappa$ when the viscous term is relatively small. In these equations $u_{n}$ and $u_{t}$ denote the normal and tangential velocity at the free surface, respectively. Furthermore $p_{0}$ denotes the ambient pressure of the air in the tank, $\sigma$ the surface tension of the fluid and $\kappa$ the mean curvature of the free surface. If the free surface is described by $S(x, y, z, t)=0$, then $\kappa$ is given by

$$
\kappa=\nabla \cdot \boldsymbol{n}=\nabla \cdot\left(\frac{\nabla S}{|\nabla S|}\right) .
$$

The dimensionless Bond number $B o$ and Weber number $W e$, defined by

$$
B o=\frac{\rho g L^{2}}{\sigma} \text { and } W e=\frac{\rho L U^{2}}{\sigma}
$$

express the relative importance of gravitational forces $(B o)$ and inertial forces $(W e)$ with respect to capillary forces, respectively. In case of high values of $B o$ and $W e$ the surface tension can be neglected. However, when the surface tension cannot be neglected, the mean curvature of the free surface has to be calculated in order to apply boundary condition (4). In this case, spatial derivatives have to be computed for equation (5) and hence boundary conditions are needed at the contact line. In this study, a static contact angle $\theta$ is prescribed, which is defined as the angle between the normal at the solid boundary and the normal at the free surface (see Figure 3). As a consequence, the free surface intersects the solid boundary at a fixed angle. The value of $\theta$ depends on the material properties of liquid, air and the solid boundary of the tank inside Sloshsat and is taken $90^{\circ}$ in this study. However, in reality the contact line probably sticks to the tank wall and hence the contact angle may not be constant. As an alternative, a dynamic contact angle in combination with contact-angle hysteresis may be applied. Recent validation of such methods, using free-fall drop-tower experiments, shows promising results. For more details and references, see Van Mourik et al. ${ }^{13}$. 


\subsection{Solid-body dynamics}

The governing equations for the Sloshsat solid-body dynamics, describing conservation of linear and angular momentum in an inertial frame, are given by

$$
\begin{aligned}
m_{s} \dot{\boldsymbol{q}}+\dot{\boldsymbol{\omega}} \times m_{s} \overline{\boldsymbol{r}}_{s}+\boldsymbol{\omega} \times\left(\boldsymbol{\omega} \times m_{s} \overline{\boldsymbol{r}}_{s}\right) & =\mathcal{F}+m_{s} \boldsymbol{F} \\
m_{s} \overline{\boldsymbol{r}}_{s} \times \dot{\boldsymbol{q}}+\boldsymbol{I}_{s} \dot{\boldsymbol{\omega}}+\boldsymbol{\omega} \times \boldsymbol{I}_{s} \boldsymbol{\omega} & =\mathcal{T}+m_{s} \overline{\boldsymbol{r}}_{s} \times \boldsymbol{F}
\end{aligned}
$$

In these equations, $m_{s}, \overline{\boldsymbol{r}}_{s}$ and $\boldsymbol{I}_{s}$ denote the mass, centre of mass and moment of inertia (MOI) of the solid body ('dry' Sloshsat), respectively, where $\overline{\boldsymbol{r}}_{s}$ and $\boldsymbol{I}_{s}$ are taken relative to the moving coordinate system. Furthermore, $\boldsymbol{\omega}$ and $\dot{\boldsymbol{\omega}}$ denote the angular velocity and acceleration of the moving frame, respectively and $\dot{\boldsymbol{q}}$ is the linear acceleration of the moving reference frame relative to the inertial frame. The vector $\boldsymbol{F}$ represents an additional acceleration due to an external force, such as the thruster induced forces on the satellite. The calligraphic symbols $\mathcal{F}$ and $\mathcal{T}$ represent the force and torque that the fluid via pressure (normal stress) and viscous effects (tangential stress) exerts on the boundary of the solid body of Sloshsat.

\subsection{Coupled solid-liquid dynamics}

The description of the interaction between the sloshing fluid inside Sloshsat and the motion of Sloshsat itself requires a coupling between the two models for fluid dynamics (Section 2.1) and solid-body dynamics (Section 2.2).

The influence of the solid-body motion on the fluid is described through the virtual body force

$$
\boldsymbol{f}=-\dot{\boldsymbol{q}}-\dot{\boldsymbol{\omega}} \times \boldsymbol{r}-\boldsymbol{\omega} \times(\boldsymbol{\omega} \times \boldsymbol{r})-2 \boldsymbol{\omega} \times \boldsymbol{u},
$$

which is used in equation (2). In first instance, the influence of the sloshing liquid on the solid body is described by means of

$$
\begin{aligned}
\mathcal{F} & =\oint_{\partial V}\left(p \boldsymbol{I}_{3}-\mu \nabla \boldsymbol{u}\right) \cdot \boldsymbol{n} d S \\
\mathcal{T} & =\oint_{\partial V}\left(\boldsymbol{r} \times\left(p \boldsymbol{I}_{3}-\mu \nabla \boldsymbol{u}\right)\right) \cdot \boldsymbol{n} d S
\end{aligned}
$$

where $\boldsymbol{I}_{3}$ is the $3 \times 3$ identity matrix and $\boldsymbol{n}$ the outward-pointing normal on the boundary $\partial V$ of the total volume $V$ of the solid body. Using the divergence theorem, these integrals can be written as integrals over the total volume $V$

$$
\begin{aligned}
\mathcal{F}=\int_{V} \nabla p-(\nabla \cdot \mu \nabla) \boldsymbol{u} d V & =-\int_{V} \rho\left(\frac{D \boldsymbol{u}}{D t}-\boldsymbol{F}-\boldsymbol{f}\right) d V \\
\mathcal{T}=\int_{V} \boldsymbol{r} \times(\nabla p-(\nabla \cdot \mu \nabla) \boldsymbol{u}) d V & =-\int_{V} \rho \boldsymbol{r} \times\left(\frac{D \boldsymbol{u}}{D t}-\boldsymbol{F}-\boldsymbol{f}\right) d V,
\end{aligned}
$$


where the Navier-Stokes equations (1) and (2) are used to deduce the expressions in terms of the material derivative

$$
\frac{D \boldsymbol{u}}{D t}=\frac{\partial \boldsymbol{u}}{\partial t}+(\boldsymbol{u} \cdot \nabla) \boldsymbol{u} .
$$

The integration is over the full volume $V$ of the solid body, i.e. both the liquid volume and the void. To make sure that the void does not contribute to the integrals, the density in the void is set to zero.

In the equations for the solid-body dynamics (6) and (7), the left-hand sides contain the mass of the solid body, while on the right-hand side the mass of the liquid appears. Intuitively, one can see that solving the system iteratively may lead to an unstable procedure when the liquid mass is too large compared to that of the solid body. Therefore, the system for the solid-body dynamics is rewritten before discretisation is applied. For a more detailed analysis of the stability behaviour of the iteration process for solving (6) and (7), see Gerrits ${ }^{14,15}$ and Vreeburg and Veldman ${ }^{9}$. The final equations for the solid-body dynamics read

$$
\begin{aligned}
m \dot{\boldsymbol{q}}+\dot{\boldsymbol{\omega}} \times \overline{\boldsymbol{r}}+\boldsymbol{\omega} \times(\boldsymbol{\omega} \times \overline{\boldsymbol{r}}) & =-\int_{V} \rho\left(\frac{D \boldsymbol{u}}{D t}+2 \boldsymbol{\omega} \times \boldsymbol{u}-\boldsymbol{F}\right) d V+m_{s} \boldsymbol{F} \\
m \overline{\boldsymbol{r}} \times \dot{\boldsymbol{q}}+\boldsymbol{I} \dot{\boldsymbol{\omega}}+\boldsymbol{\omega} \times \boldsymbol{I} \boldsymbol{\omega} & =-\int_{V} \rho \boldsymbol{r} \times\left(\frac{D \boldsymbol{u}}{D t}+2 \boldsymbol{\omega} \times \boldsymbol{u}-\boldsymbol{F}\right) d V+m_{s} \overline{\boldsymbol{r}}_{s} \times \boldsymbol{F}
\end{aligned}
$$

In these equations, $m=m_{s}+m_{l}$ is the total mass (solid+liquid) of Sloshsat, $\boldsymbol{I}=\boldsymbol{I}_{s}+\boldsymbol{I}_{l}$ is the MOI of the coupled system and $\overline{\boldsymbol{r}}=\left(m_{s} \overline{\boldsymbol{r}}_{s}+m_{l} \overline{\boldsymbol{r}}_{l}\right) / m$ the centre of mass of the coupled system. Since the mass of the liquid (which appears on the right-hand side of the equations) will always be smaller than the total mass of the coupled system (on the left-hand side), the system (11) and (12) can be solved iteratively in a stable manner.

\section{THE NUMERICAL MODEL}

\subsection{Discretisation of the liquid model}

Since the Navier-Stokes equations cannot be solved analytically, the equations are solved by means of numerical techniques. A rectangular Cartesian grid is used that widely covers the flow domain, as this type of grid is easy to construct and makes finitevolume discretisation of the conservation equations (1) and (2) straightforward. Moreover, much research has been done on numerical tracking of free surfaces on orthogonal grids (Gerrits ${ }^{14}$, Kleefsman et al. ${ }^{11}$ ).

A disadvantage of a rectangular grid is that the curved solid boundary of the water tank inside Sloshsat is not aligned with the computational cell faces. Avoiding staircase-type geometries, the solid boundary must be allowed to cut through the grid cells arbitrarily. In order to recognize the flow domain, in every grid cell a volume aperture $F^{b}$ and edge apertures $A^{x}, A^{y}$ and $A^{z}$ are introduced, indicating the fraction of a cell or cell face that is open for flow. Apart from these geometry apertures, also a free-surface aperture $F^{s}$ is 


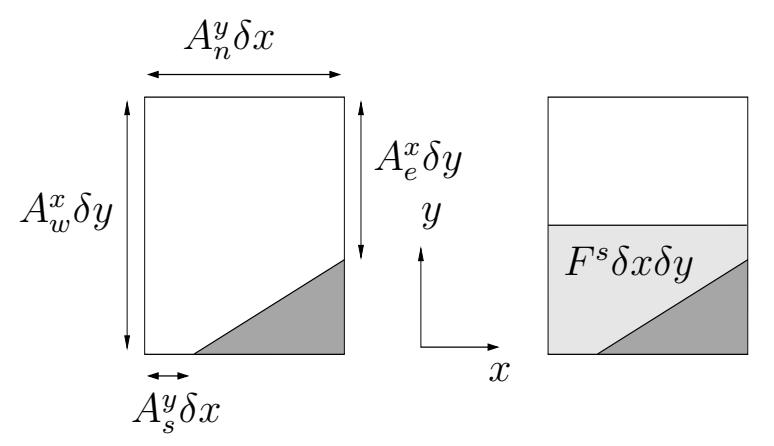

Figure 4: Two dimensional illustration of the volume and edge apertures for the geometry (left) and the volume aperture for the free surface (right). Dark and light shading represent solid body and liquid, respectively.

introduced, indicating the fraction of a cell that is actually filled with fluid. This aperture is also known as the volume-of-fluid (VOF) function (Hirt and Nichols ${ }^{16}$ ). In Figure 4, a two-dimensional illustration of these apertures is given, where $\delta x$ and $\delta y$ denote the mesh size in $x$ - and $y$-direction, respectively, and $n, s, e$ and $w$ refer to north, south, east and west, respectively. The volume apertures for the geometry and free-surface are naturally related by $0 \leq F^{s} \leq F^{b} \leq 1$.

For the spatial discretisation of equations (1) and (2), the finite-volume method (FVM) is applied. In this method, the equations are formulated in integral conservation form for an arbitrary control volume. Subsequently, the computational domain is subdivided in control volumes. For each computational cell, surface integrals over the boundary faces of the convection and diffusion fluxes are evaluated. These fluxes are written in terms of the discrete values of variables in neighbouring cells by means of interpolation, see e.g. Luppes ${ }^{17}$. Verstappen and Veldman ${ }^{18}$ have shown that the stability of the numerical scheme is enhanced when the interpolation is chosen carefully and the discretised equation has the same symmetry properties as the continuous equation. Because of the use of the conservation principle, the FVM has a physical meaning. The discrete conservation in a certain control volume is equivalent to the continuous conservation that underlies the derivation of the original conservation equation.

The continuity equation (1) is discretised for the computational cell shown in Figure 5 , making use of the apertures describing the part of the cell faces that are open for flow. Discrete conservation of mass implies that the sum of all mass fluxes through the boundary $\partial V$ of the computational cell should vanish. For the computational cell in Figure 5 this results in

$$
u_{e} A_{e}^{x} \delta y+v_{n} A_{n}^{y} \delta x-u_{w} A_{w}^{x} \delta y-v_{s} A_{s}^{y} \delta x=0,
$$

where some notation of Figure 4 is used and the mass flux through the solid boundary is taken zero. The result is a second-order discretisation of the continuity equation (1). The spatial discretisation of the convection, diffusion and pressure terms in equation (2) is obtained in a similar way. For more details, see Gerrits $^{14}$, Fekken ${ }^{12}$ or Kleefsman et al. ${ }^{11}$. 


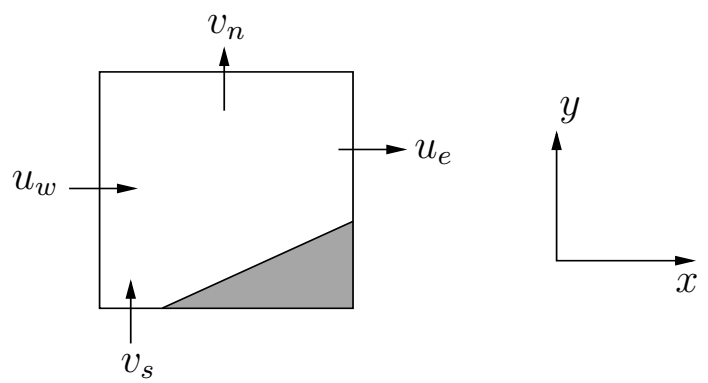

Figure 5: Two dimensional computational cell with staggered velocities. The shading represents solid body.

At the solid boundary the no slip condition $\mathbf{u}=0$ is applied. In the neighbourhood of the free surface, boundary condition (3) is applied for velocity components between two adjacent empty cells. Between an empty cell and a cell (partly) filled with fluid, velocity components are found by means of interpolation based on mass conservation.

For the temporal derivatives in equations (1) and (2) the forward Euler method is used. This results in

$$
\begin{aligned}
\nabla \cdot \boldsymbol{u}^{(n+1)} & =0 \\
\frac{\boldsymbol{u}^{(n+1)}-\boldsymbol{u}^{(n)}}{\delta t}+\left(\boldsymbol{u}^{(n)} \cdot \nabla\right) \boldsymbol{u}^{(n)} & =-\frac{1}{\rho} \nabla p^{(n+1)}+\frac{\mu}{\rho} \Delta \mathbf{u}^{(n)}+\boldsymbol{F}^{(n)}+\boldsymbol{f}^{(n)},
\end{aligned}
$$

where the superscript indicates the time level and $\delta t$ denotes the time step. The combination of temporal and spatial discretisation leads to

$$
\begin{aligned}
\mathcal{M} \boldsymbol{u}_{h}^{(n+1)} & =0 \\
\mathcal{V} \frac{\boldsymbol{u}_{h}^{(n+1)}-\boldsymbol{u}_{h}^{(n)}}{\partial t}+\mathcal{C}\left(\boldsymbol{u}_{h}^{(n)}\right) \boldsymbol{u}_{h}^{(n)} & =-\frac{1}{\rho} \mathcal{P} \boldsymbol{p}_{h}^{(n+1)}+\frac{\mu}{\rho} \mathcal{D} \boldsymbol{u}_{h}^{(n)}+\boldsymbol{F}_{h}^{(n)}+\boldsymbol{f}_{h}^{(n)}
\end{aligned}
$$

in which $\boldsymbol{u}_{h}, \boldsymbol{p}_{h}, \boldsymbol{F}_{h}$ and $\boldsymbol{f}_{h}$ are vectors containing all discrete velocities, pressures and forces, respectively, and $\mathcal{M}, \mathcal{V}, \mathcal{P}, \mathcal{C}$ and $\mathcal{D}$ denote coefficient matrices. The matrix $\mathcal{V}$ is a diagonal matrix containing cell volumes. The matrices $\mathcal{M}$ and $\mathcal{P}$ contain geometrical information, where each row of $\mathcal{M}$ corresponds to the discrete continuity equation in a computational cell. Analytically, the divergence operator $\nabla \cdot$ in (14) and gradient operator $\nabla$ in $(15)$ are related by $\nabla=-(\nabla \cdot)^{T}$. In the discretised equations this property is preserved by means of $\mathcal{P}=-\mathcal{M}^{T}$, see e.g. Gerrits ${ }^{14}$. The matrices $\mathcal{C}$ and $\mathcal{D}$ correspond to the discretised convective and diffusive operators. Note that besides geometrical information, the matrix $\mathcal{C}$ contains velocities as well. Verstappen and Veldman ${ }^{18}$ have shown that the stability of the numerical scheme is enhanced when the symmetry properties of the continuous equation are preserved numerically. Hence, $\mathcal{C}$ and $\mathcal{D}$ should be skewsymmetric and symmetric matrices, respectively, like the continuous analytical convective and diffusive operators. 
Combination of equations (16) and (17) leads to the Poisson equation for the pressure $\boldsymbol{p}_{h}^{(n+1)}$

$$
-\mathcal{M} \mathcal{V}^{-1} \mathcal{M}^{T} \boldsymbol{p}_{h}^{(n+1)}=\frac{\rho}{\delta t} \mathcal{M} \tilde{\boldsymbol{u}}_{h}^{(n)}
$$

in which the so called intermediate velocity field $\tilde{\boldsymbol{u}}_{h}$ is defined as

$$
\tilde{\boldsymbol{u}}_{h}=\boldsymbol{u}_{h}^{(n)}+\delta t \mathcal{V}^{-1}\left(-\mathcal{C}\left(\boldsymbol{u}_{h}^{(n)}\right) \boldsymbol{u}_{h}^{(n)}+\frac{\mu}{\rho} \mathcal{D} \boldsymbol{u}_{h}^{(n)}+\boldsymbol{F}_{h}^{(n)}+\boldsymbol{f}_{h}^{(n)}\right) .
$$

The Poisson matrix in the left-hand side of (18) depends on geometrical information only, whereas the right-hand side depends on velocities, $\delta t, \rho, \mu, \boldsymbol{F}, \boldsymbol{f}$ and mesh sizes. At the free surface, the boundary condition (4) for the pressure is included by means of interpolation in such a way that the Poisson matrix is always diagonally dominant. The linear equation (18) is solved iteratively using a SOR method with automatically adjusted relaxation parameter for optimal convergence behaviour $\left(\right.$ Botta $\left.^{19}\right)$. Once the pressure $\boldsymbol{p}_{h}^{(n+1)}$ at the new time level is determined, it is subsequently substituted in equation (17) to obtain the new velocity field $\boldsymbol{u}_{h}^{(n+1)}$.

\subsection{Free-surface displacement}

When the new velocity field at time level $(n+1)$ is known, the free surface can be displaced. This is done using an adapted version of the VOF method introduced by Hirt and Nichols ${ }^{16}$.

Making use of the apertures, every grid cell can be given a label. A distinction is made between five different types of computational cells. The interior cells containing no fluid, i.e. $F^{b}>0$ and $F^{s}=0$, are labeled as $\mathrm{E}$ (mpty) cells. Non-empty cells $\left(F^{s}>0\right)$ adjacent to $\mathrm{E}$ cells are labeled as $\mathrm{S}$ (urface) cells, as they must contain part of the free surface. All the remaining non-empty cells are labeled $\mathrm{F}$ (ull) cells. Cells satisfying $F^{s}=F^{b}=0$ are called $\mathrm{B}$ (oundary) cells when they are adjacent to an interior cell, otherwise they are labeled as $\mathrm{e}(\mathrm{X})$ terior. In Figure 6 an example of a label configuration is shown.

The displacement of the free surface is achieved by the advection of the VOF-function $F^{s}$ in time, which satisfies the (continuity) equation

$$
\frac{\partial F^{s}}{\partial t}+(\mathbf{u} \cdot \nabla) F^{s}=0
$$

At each cell, the amount of fluid that is transported from one computational cell (donor) to another (acceptor) depends on the magnitude of velocity, time step and grid sizes. As an example, the flux $\delta F_{e}^{s}$ across the eastern cell face in positive $x$-direction is given by

$$
\delta F_{e}^{s}=u \delta t A_{e}^{x} \delta y
$$

where some notation of Figure 4 is adopted. When for each cell face the flux is known, the VOF function at the new time level $(n+1)$ is given by

$$
F^{b} \delta x \delta y F^{s(n+1)}=F^{b} \delta x \delta y F^{s(n)}-\delta F_{e}^{s}+\delta F_{w}^{s}-\delta F_{n}^{s}+\delta F_{s}^{s} .
$$




\begin{tabular}{|c|c|c|c|c|c|}
\hline $\mathrm{E}$ & $\mathrm{E}$ & $\mathrm{E}$ & $\mathrm{E}$ & $\mathrm{E}$ & $\mathrm{E}$ \\
\hline $\mathrm{S}$ & $\mathrm{E}$ & $\mathrm{E}$ & $\mathrm{E}$ & $\mathrm{S}$ & $\mathrm{S}$ \\
\hline $\mathrm{F}$ & $\mathrm{S}$ & $\mathrm{S}$ & $\mathrm{S}$ & $\mathrm{F}$ & $\mathrm{F}$ \\
\hline $\mathrm{X}$ & $\mathrm{B}$ & $\mathrm{F}$ & $\mathrm{F}$ & $\mathrm{F}$ & $\mathrm{B}$ \\
\hline $\mathrm{X}$ & $\mathrm{B}$ & $\mathrm{F}$ & $\mathrm{F}$ & $\mathrm{F}$ & $\mathrm{B}$ \\
\hline
\end{tabular}

Figure 6: Two dimensional grid-cell labeling for an arbitrary geometry and liquid configuration. Dark and light shading represent solid body and liquid, respectively.

However, near the free surface, the cells are generally not completely filled with liquid. In that case, the flux may exceed the amount available in the donor cell or the void present in the acceptor cell. Therefore, the actual fluxes used are modified, based on the local contents of the neighbouring cells (Hirt and Nichols ${ }^{16}$, Gerrits ${ }^{14}$ ). An important drawback of the original donor-acceptor method is the creation of unrealistic small drops of fluid that get separated from the bulk fluid. Moreover, $F^{s}$ values may be outside the range $0 \leq F^{s} \leq F^{b}$, even after the modification of the fluxes. Therefore, in the present study, an additional correction in terms of a local height function is used that is based on the orientation of the free surface $\left(\right.$ Gerrits $^{14}$, Kleefsman et al. $\left.{ }^{11}\right)$. For every surface cell a function is defined that gives the height of the fluid in a column of three cells. The direction in which the function is defined is the coordinate direction that is most normal to the free surface, as shown in Figure 7. After computing the fluxes across the cell faces of all three cells, the height function is updated and the individual $F^{s}$ values are subsequently reconstructed from the modified fluid height in the column.

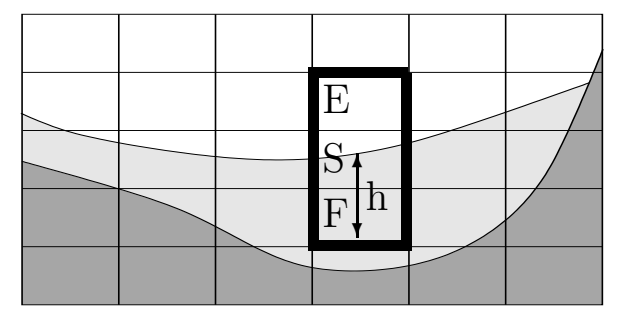

Figure 7: Definition of the height function $h$ for a surface cell $\mathrm{S}$ in an arbitrary geometry and liquid configuration. Dark and light shading represent solid body and liquid, respectively.

An alternative method that is widely used, which is however far more difficult to implement, has been proposed by Youngs ${ }^{20}$. Both methods have been compared recently by Kleefsman et al. ${ }^{11}$, leading to the conclusion that for certain flow applications Youngs' method yields slightly better results. However, for the Sloshsat simulations in the present study, the donor-acceptor method with local height function leads to satisfactory results. 


\subsection{Discretisation of the solid-body model}

The discretisation of equations (11) and (12) can be given schematically by means of

$$
\begin{aligned}
m \frac{d \boldsymbol{q}}{d t}^{(n+1)}+\frac{d \boldsymbol{\omega}}{d t}^{(n+1)} \times m \overline{\boldsymbol{r}}^{(n+1)} & =\mathcal{L}^{(n)} \\
m \overline{\boldsymbol{r}}^{(n+1)} \times \frac{d \boldsymbol{q}}{d t}^{(n+1)}+\boldsymbol{I}^{(n+1)} \frac{d \boldsymbol{\omega}}{d t}^{(n+1)} & =\mathcal{A}^{(n)},
\end{aligned}
$$

where the superscript again denotes the time level. The symbols $\mathcal{L}^{(n)}$ and $\mathcal{A}^{(n)}$ on the right-hand side contain $\boldsymbol{q}^{(n)}, \boldsymbol{w}^{(n)}$, as well as the fluid velocities $\boldsymbol{u}^{(n+1)}$ in the tank and the thruster forces $\boldsymbol{F}^{(n+1)}$. The temporal derivatives are integrated from time level $(n)$ to $(n+1)$ using a fourth order Runge-Kutta method. Equations (22) and (23) lead to a linear system for six unknowns (three components of both $\dot{\boldsymbol{q}}$ and $\dot{\boldsymbol{\omega}}$ ), which is solved by means of Gaussian elimination during each Runge-Kutta step, see Gerrits ${ }^{14,15}$.

\subsection{Solution procedure}

The simulation of a Sloshsat manoeuvre is started with a lasting period of prescribed rotation $\boldsymbol{\omega}$ around the stable axis of maximum MOI and zero linear acceleration $\boldsymbol{q}$, without any thruster action. In this initial period, the water inside the tank can settle towards a certain stable configuration. Only the discretised conservation equations (16) and (17) and the fluid displacement (21) are solved, as $\boldsymbol{\omega}$ and $\boldsymbol{q}$ are constant in this period. An example of the initial fluid configuration inside the Sloshsat tank is given in Figure 8. The final state after the initial period is referred to as $t=0$.

At $t=0$, the manoeuvre is started. From this time, all discretised equations are solved in the sequential order $(16)+(17),(21),(22)+(23)$, after which the next time level is reached. At each time level, the influence of the thruster forces is incorporated when solving $(22)+(23)$. By using small time steps, a sufficiently accurate time advancement procedure is thus obtained and hence no iteration at the time levels is required.

\section{EXPERIMENTS AND SIMULATIONS}

\subsection{Experiments with Sloshsat}

During a period of 8 days, several experiments have been carried out with Sloshsat in an orbit around earth. These experiments were controlled by means of 12 thrusters that could be fired with a frequency of $30 \mathrm{~Hz}$. The status of each thruster (whether or not a thruster has been fired) was recorded. The thruster forces that operate on Sloshsat can be determined subsequently from the measured pressure inside the fuel tank. The various experiments and measurement equipment are extensively described by Vreeburg ${ }^{5-7}$.

In the present study, three kinds of experiments are considered. First, simple spin-up and spin-down is considered, where the rotational velocity $\boldsymbol{\omega}$ around the axis of maximum MOI is increased or decreased. This is achieved by firing two thrusters that form a couple. 

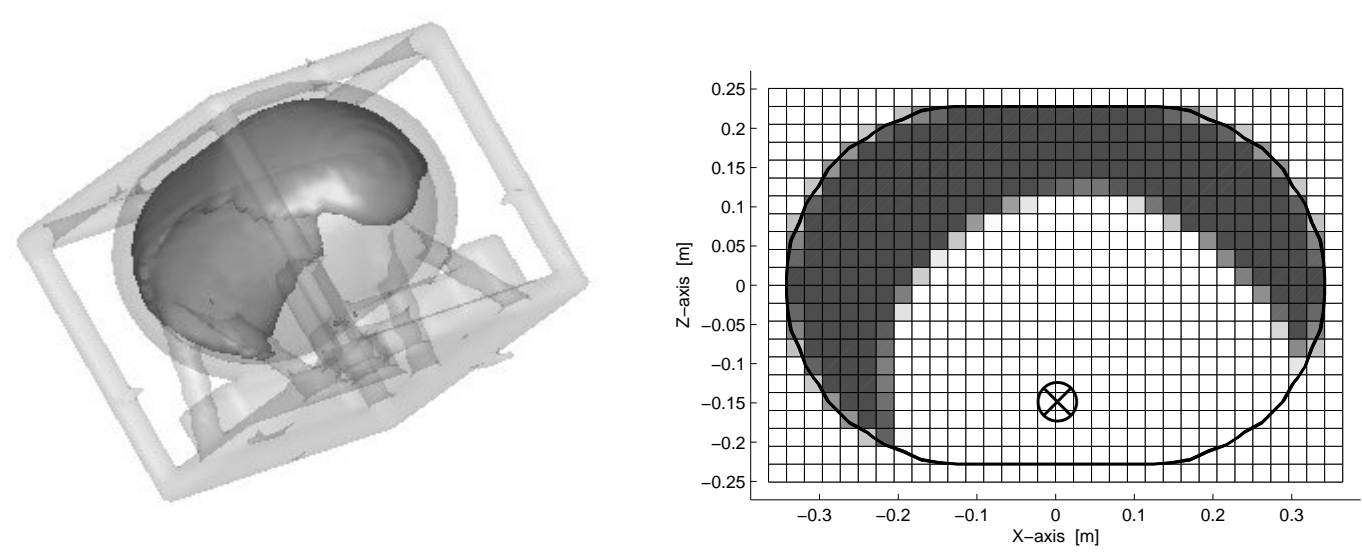

Figure 8: The initial Sloshsat fluid configuration $(t=0)$ after prescribed rotation. In the right figure a 2D slice is given, where dark and light shading represent water and air, respectively, based on the numerical value of $F^{s}$ in the numerical grid. The rotation axis is indicated by $\otimes$.

Next, nutation-avoidance manoeuvres (NAM) are considered, where the objective of the experiment is to move the angular rotation vector without incurring too much nutation. This is achieved by a series of specific thruster actions that depends on the rotational rate, specially designed to cancel liquid slosh. A practical example is an observational satellite that has to be pointed towards another direction. In such a case, heavy nutation in combination with a low level of damping is very undesirable.

The third kind of experiment deals with large-scale fluid motion. In these so-called flat-spin experiments, Sloshsat is forced to rotate around the axis of intermediate MOI for some time, during which the fluid configuration adjusts. After some time, the thruster action is canceled and a free tumble of Sloshsat commences. During this free tumble, the rotational direction of Sloshsat slowly moves towards the maximum MOI with a lot of nutation and fluid action. Hence, such a manoeuvre is very suitable for the validation of the numerical model.

\subsection{Comparison of measurements and simulations}

The first experiment considered is a spin-up where the rotational velocity changes from $\boldsymbol{\omega}=[-0.004 ; 0.104 ;-0.007]^{T}$ towards a rotation with $\boldsymbol{\omega}_{y} \approx 0.179$ and nutating $\boldsymbol{\omega}_{x}$ and $\boldsymbol{\omega}_{z}$ components. In Figure 9, measurements are compared with the results of a simulation. The increase from $\boldsymbol{\omega}_{y} \approx 0.104$ to $\boldsymbol{\omega}_{y} \approx 0.179$ is almost perfect. However, the results of $\boldsymbol{\omega}_{x}$ and $\boldsymbol{\omega}_{z}$ show a clear difference between simulation and experiment. Although the frequency of the nutation in $\boldsymbol{\omega}_{x}$ and $\boldsymbol{\omega}_{z}$ is comparable, in the simulation the amplitudes are too small. A proper calibration of the measured data may yield a favourable correction of the measured values of $\boldsymbol{\omega}$, especially with respect to the small values of $\boldsymbol{\omega}_{x}$ and $\boldsymbol{\omega}_{z}$. The decrease of the amplitude is too strong in the simulation. The 

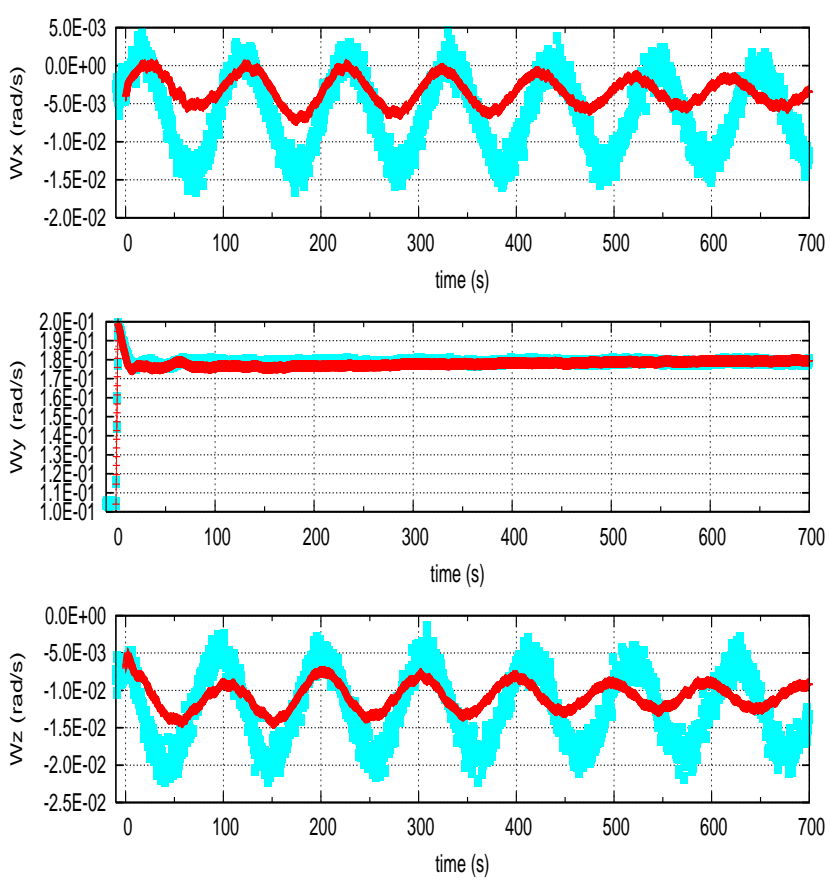

experiment $\quad$ simulation ++

Figure 9: The comparison of numerical simulation with measurements for the spin-up experiment. In the three subfigures, the rotational velocity components $\boldsymbol{\omega}_{x}$ (upper), $\boldsymbol{\omega}_{y}$ (middle) and $\boldsymbol{\omega}_{z}$ (lower) are depicted. The simulation is represented by the darker lines.

high level of damping in the simulations is probably mainly caused by the use of first-order spatial discretisation to enhance stability. This generally results in a high level of nonphysical (numerical) diffusion, depending on the magnitude of fluid velocity. Capillary forces are of less importance for the observed damping, as the magnitude of $\boldsymbol{\omega}$ is relatively large in this experiment.

The comparison between simulation and experiment for a spin-down experiment is depicted in Figure 10. In the experiment, the rotational velocity changes from $\boldsymbol{\omega}=$ $[-0.006 ; 0.179 ;-0.013]^{T}$ towards $\boldsymbol{\omega}_{y} \approx 0.128$, with nutating $\boldsymbol{\omega}_{x}$ and $\boldsymbol{\omega}_{z}$ components. The decrease of $\boldsymbol{\omega}_{y}$ as a result of the thruster action in the first few seconds shows a reasonable agreement. After some time $(t=200 s)$, the simulation overpredicts the value of $\boldsymbol{\omega}_{y}$. The level of damping in $\boldsymbol{\omega}_{x}$ and $\boldsymbol{\omega}_{z}$, obtained in the simulation, is lower than in the spin-up experiment. The magnitude of $\boldsymbol{\omega}$, and hence that of the fluid velocity, is smaller in this case, which causes only a moderate level of numerical diffusion. The amplitude of $\boldsymbol{\omega}_{x}$ of the simulation is too large initially, whereas that of $\boldsymbol{\omega}_{z}$ is comparable. The obtained nutation frequencies are somewhat higher in the simulation. A proper calibration of the measured data may yield a favourable correction of the small measured values of $\boldsymbol{\omega}_{x}$ and 

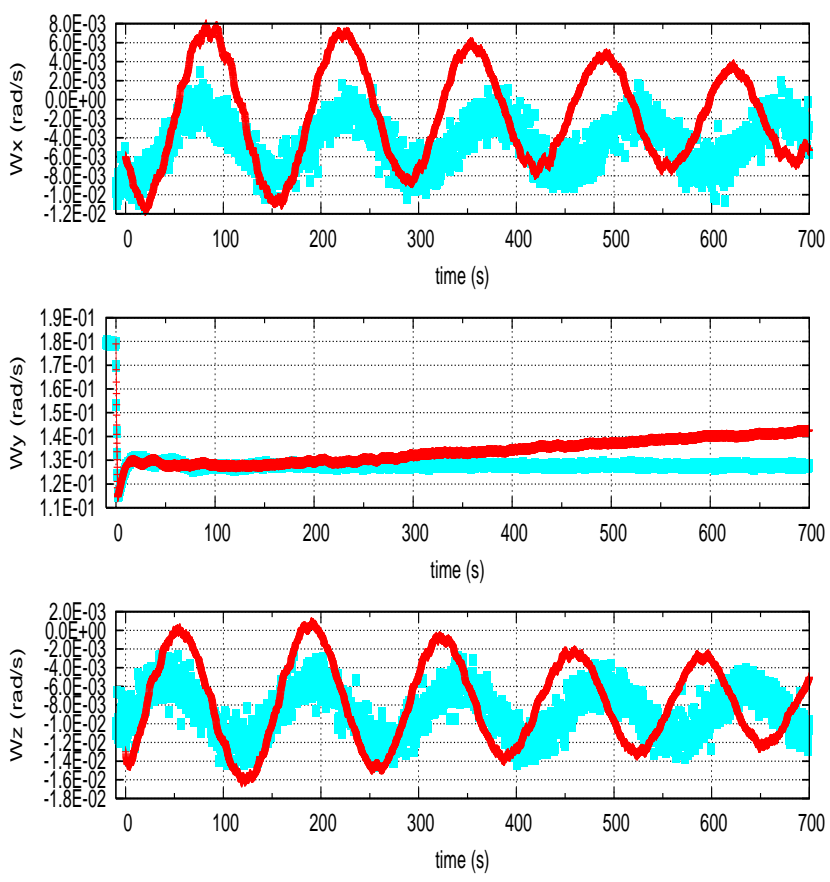

experiment $\quad$ simulation ++

Figure 10: The comparison of numerical simulation with measurements for the spin-down experiment. The simulation is represented by the darker lines.

$\boldsymbol{\omega}_{z}$. Improved agreement between experiment and simulation may probably be obtained by the use of a dynamic contact angle in the computational model, since capillary forces are rather important at the low rotational velocity of this experiment.

In Figure 11, a NAM experiment is compared with a numerical simulation. The manoeuvre starts with an adapted fluid configuration at $\boldsymbol{\omega}=[0.003 ;-0.101 ; 0.007]^{T}$. In the $\boldsymbol{\omega}_{x}$ component (top figure), the series of thruster pulses can be distinguished. At $t=0 \mathrm{~s}$, $t=70 \mathrm{~s}$ and $t=140 \mathrm{~s}, \boldsymbol{\omega}_{x}$ decreases as a direct result of a short period of thruster action, followed by periods of free-tumble movement. The simulation is in good agreement with the measurements of $\boldsymbol{\omega}_{x}$ and $\boldsymbol{\omega}_{z}$, with comparable nutation frequencies and amplitudes. After $t=300 \mathrm{~s}$, the $\boldsymbol{\omega}_{y}$ component starts to deviate. Since the rotational speed is low and the NAM only induces small scale liquid motion, the level of numerical diffusion is small, resulting in a comparable level of damping. As the rotational velocity at this experiment is rather small and hence capillary forces are rather important, further improvement may be obtained by the use of a dynamic contact-angle model.

The comparison for the flat-spin manoeuvre is given in Figure 12. The manoeuvre starts with a fluid configuration that is adapted to $\boldsymbol{\omega}=[-0.005 ; 0.165 ;-0.011]^{T}$. In the first 14 seconds, Sloshsat is approximately rotating around around the axis of intermediate MOI. 

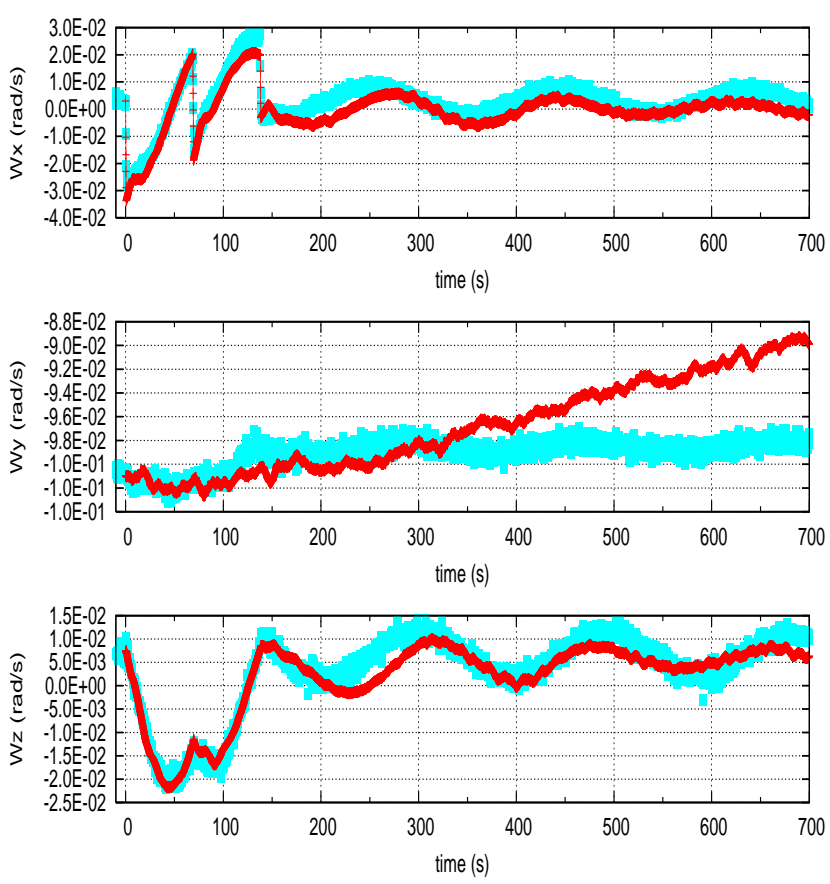

experiment $\quad$ simulation ++

Figure 11: The comparison of numerical simulation with measurements for the NAM experiment. The simulation is represented by the darker lines.

After this period, the thruster action continues until $t=33 \mathrm{~s}$. The obtained components of $\boldsymbol{\omega}$ of the simulation are in good agreement with those of the measurements, except for the high level of damping. The flat-spin induces a lot of fluid motion and liquid sloshing, with rather high values of the velocity. Hence, in the numerical simulation, the effect of numerical diffusion is clearly visible. The profile of $\boldsymbol{\omega}_{y}$ shows an interesting phenomenon. Each nutation period contains a higher frequency, which is the result of sloshing water pounding the tank wall. This secondary frequency is present in both experiment and simulation, indicating that in the simulation the Sloshsat motion adopts the sloshing frequency correctly. The Sloshsat orientation and water configuration during the simulation of the flat-spin manoeuvre is depicted in Figure 13. In the first subfigure, the water inside Sloshsat is adapted to the rotation around the axis of intermediate MOI $(t=14 s)$. In the next figures, the fluid movement during the free tumble manoeuvre is shown. In the two final subfigures, the transition toward stable rotation around the axis of maximum MOI is almost completed $(t=830 \mathrm{~s}$ and $831 \mathrm{~s})$. The orientation in these subfigures indicate a rotation period of $T \approx 12$ seconds, which is in agreement with $\boldsymbol{\omega}_{y} \approx-0.55$ (Figure 12). 

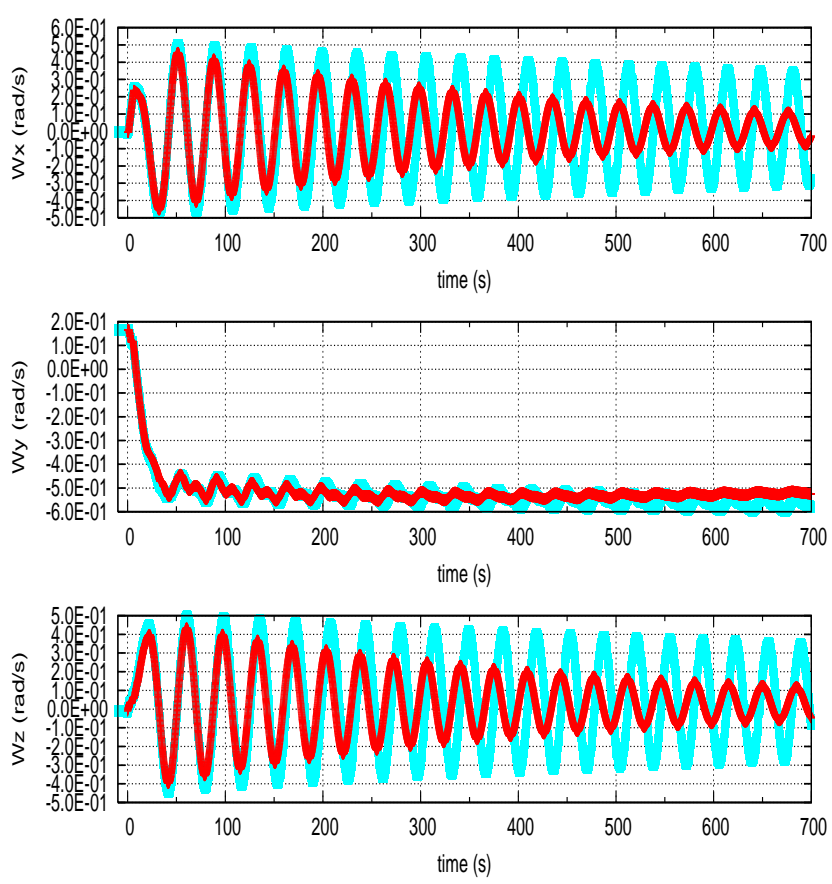

experiment $\quad$ simulation +

Figure 12: The comparison of numerical simulation with measurements for the flat-spin experiment. The simulation is represented by the darker lines.

\section{CONCLUSIONS}

In this study, the influence of sloshing liquid on board spacecraft and satellites is investigated, as liquid management and its influence on the overall spacecraft dynamics is becoming increasingly important. Experiments have been carried out with the satellite 'Sloshsat FLEVO' in an orbit around earth. These experiments were supported by a theoretical/computational model based on the Navier-Stokes equations for 3D incompressible free-surface flow. This model includes capillary surface physics as well as coupled solid-liquid interaction dynamics.

Measurements of the rotational velocity of Sloshsat are compared with numerical simulations for three kinds of experiments. These experiments include both small-scale and large-scale liquid motion. The obtained frequencies in angular velocities are reasonably comparable at various rotational rates during various satellite manoeuvres. This means that in the numerical model, the sloshing frequency of the water inside the tank is adopted correctly by the satellite motion. The damping of the nutation amplitudes, observed in the simulations, is too large, as a result of additional numerical diffusion in the computational model. As this effect depends on the magnitude of fluid velocity, it is most visible at 


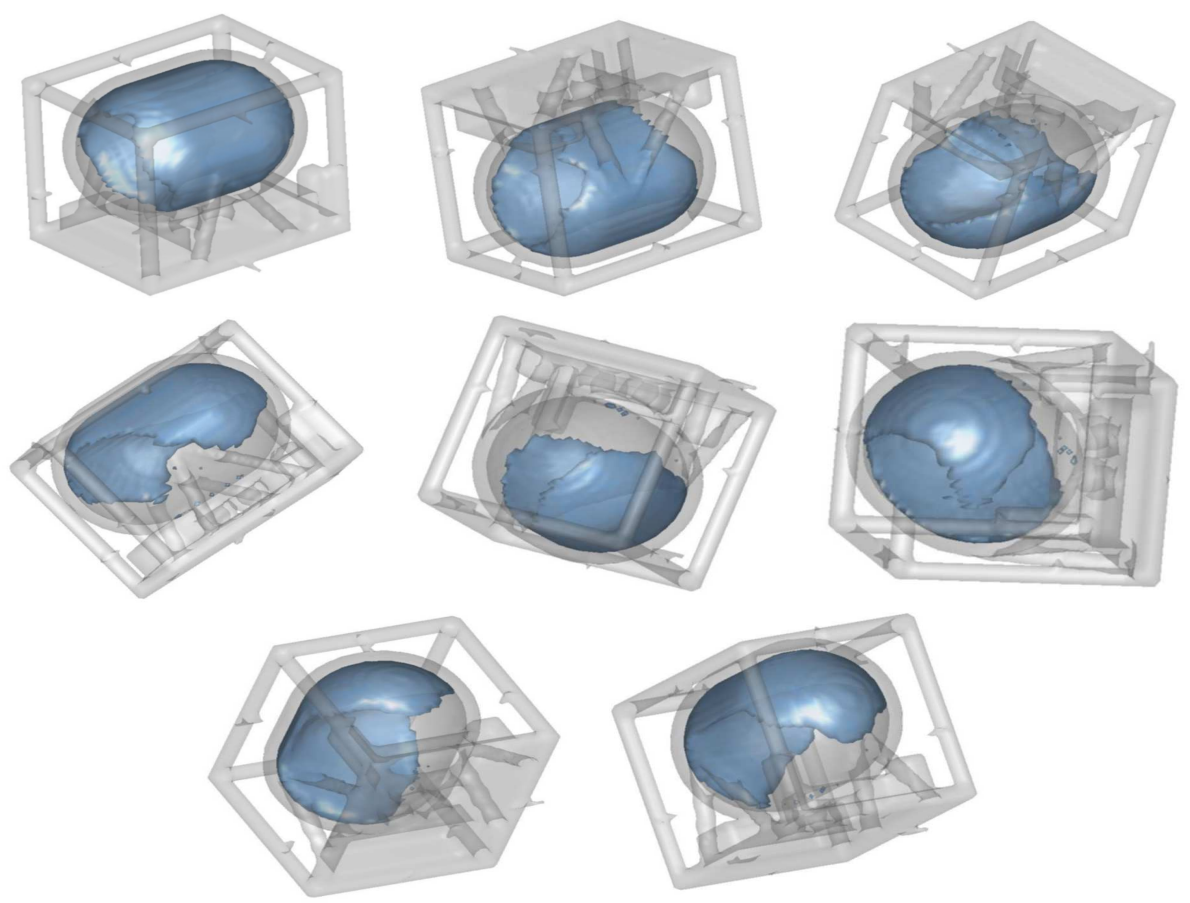

Figure 13: Sloshsat orientation and water configuration during a flat-spin manoeuvre at $t=14,18,32$, $37,44,64,830$ and 831 seconds. (lexicographical order).

experiments with large rotational velocities and fluid velocities. The use of higher-order discretisation techniques that reduce the level of numerical diffusion is recommended.

At low rotational rates and small-scale liquid motion, capillary effects are important for the obtained damping of manoeuvre-induced oscillations in the angular velocities. In case of large scale liquid motion, such as obtained during flat-spin manoeuvres, capillary forces are less important. In the present numerical model, a constant contact angle is assumed. However, in reality the contact line probably sticks to the tank wall, which means that there is less fluid motion and hence less viscous damping in Sloshsat in comparison with the simulations. The use of a dynamic contact angle in the numerical model probably yields a better agreement between simulation and experiments at low rotational velocities and is recommended. A proper calibration of the small components of $\boldsymbol{\omega}$ may yield a favourable correction of the measured values.

\section{Acknowledgement}

The research was funded by the National Institute for Space Research in the Netherlands (SRON), project nr. MG-065 


\section{REFERENCES}

[1] Strikwerda, T.E., Ray, J.C., Haley, D.R. and O'Shaughnessy, D.J. NEAR Shoemaker: major anomaly survival, delayed rendezvous and mission success. In Guidance and Control 2001, volume 107 Advances in the Astronautical Sciences, pages 597-614. American Astronautical Society, 2001.

[2] NEAR homepage, The. Final report of the NEAR anomaly review board, 1998. http://near.jhuapl.edu.

[3] NASA homepage, The. The Apollo 11 lunar surface journal, 2006. http://www.history.nasa.gov/alsj/a11/a11.landing.html.

[4] NLR Homepage, The. Sloshsat flyer http://www.nlr.nl/public/publications/ pdf/f183-02.pdf.

[5] VReeburg, J.P.B. Measured states of sloshsat Flevo. In 56th International Astronautical Congress. International Astronautical Federation, 2005. IAF-05-C1.2.09.

[6] VReeburg, J.P.B. Sloshsat Flevo experiment definition document (edd). Technical Report CR 96755 L, NLR, 1996.

[7] VReeburg, J.P.B. Diagnosis of water motion in the sloshsat FLEVo tank. In 50th International Astronautical Congress. International Astronautical Federation, 1999. IAF-99-J.2.04.

[8] Prins, J.J.M. Sloshsat FlEvo: Facility for liquid experimentation and verification in orbit. In 51st International Astronautical Congress. International Astronautical Federation, 2000. IAF-00-J.2.05.

[9] Vreeburg, J.P.B. and Veldman, A.E.P. Transient and sloshing motions in an unsupported container. In R. Monti, editor, Physics of Fluids in Microgravity, pages 293-321. Gordon and Breach Academic Publishers, 2002.

[10] Veldman, A.E.P and Vogels, M.E.S. Axisymmetric liquid sloshing under low gravity conditions. Acta Astronautica, 11:641-649, 1984.

[11] Kleefsman, K.M.T., Fekken, G., Veldman, A.E.P., Iwanowski, B. and Buchner, B. A volume-of-fluid based simulation method for wave impact problems. J. Comput. Physics, 206:363-393, 2005.

[12] Fekken, G. Numerical Simulation of Free-Surface Flow with Moving Rigid Bodies. PhD thesis, University of Groningen, The Netherlands, 2004.

[13] Van Mourik, S., Veldman, A.E.P and Dreyer, M.E. Simulation of capillary flow with a dynamic contact angle. Microgravity Sci. Techn., 17-3:91-98, 2005.

[14] Gerrits, J. Dynamics of Liquid-Filled Spacecraft. PhD thesis, University of Groningen, The Netherlands, 2001.

[15] Gerrits, J. and Veldman, A.E.P. Dynamics of liquid-filled spacecraft. J. Eng. Math., 45:21-38, 2003.

[16] HirT, C.R. and Nichols, B.D. Volume of fluid (vof) method for the dynamics of free boundaries. J. Comput. Physics, 39:201-225, 1981.

[17] Luppes, R. The Numerical Simulation of Turbulent Jets and Diffusion Flames. PhD thesis, Eindhoven University of Technology, The Netherlands, 2000. 
[18] Verstappen, R.W.C.P. and Veldman, A.E.P. Symmetry-preserving discretisation of turbulent flow. J. Comput. Physics, 187:343-368, 2003.

[19] Botta, E.F.F. and Ellenbroek, M.H.M. A modified sor method for the Poisson equation in unsteady free-surface flow calculations. J. Comput. Physics, 60:119-134, 1985.

[20] Youngs, D.L. An interface tracking method for a 3D Eulerian hydrodynamics code. Technical Report AWRE/44/92/35, Atomic Weapens Research Establishment, 1987. 